\title{
Application of an electronic nose to detect head and neck cancer from exhaled breath
}

Witt K., Department of Medical Engineering, University of Applied Sciences Jena, Germany, Katharina.Witt@fhjena.de

Inhestern J., Department of Otorhinolaryngology, Friedrich-Schiller-University of Jena, Germany, Johanna.Inhestern@med.uni-jena.de

Guntinas-Lichius O., MD, Department of Otorhinolaryngology, Friedrich-Schiller-University of Jena, Germany, Orlando.Guntinas@med.uni-jena.de

Voss A., Department of Medical Engineering, University of Applied Sciences Jena, Germany, Andreas.Voss@fhjena.de

\section{Introduction}

The human exhaled breath consists of different volatile organic compounds (VOC). These VOCs are partly products of metabolic processes and can be used as biomarkers for various diseases. An electronic nose system is a technical device for the investigation of volatile compounds by using a specific gas sensor array in combination with an intelligent data analysis.

We hypothesis that primary head and neck cancer (HNC) causes an impaired metabolism leading to altered VOCs. These metabolic changes should be detectable applying an electronic nose system to investigate the exhaled breath from patients with HNC.

\section{Methods}

In this study 10 patients with primary HNC and 13 matched control subjects (REF) were enrolled. All patients and REF were male and smokers. A single exhaled breath was collected in a special developed non-gassing sampling bag by using a mouthpiece. The sensor chip is embedded in the sampling bag and is based on a metal oxide gas sensor with three different sensitive layers. Measuring time was $35 \mathrm{~min}$ for every person.

For analyzing the sensor signals the principal component analysis (PCA) is used in combination with discriminant function analysis (DA) to discriminate between the patients and REF.

\section{Results}

The exhaled breath between HNC patients and REF could be separated with an accuracy of $82.3 \%$ by quadratic discriminant analysis using the $1^{\text {st }}$ and $2^{\text {nd }}$ principal odour compound.

\section{Conclusion}

The results of this pilot study suggest that an electronic nose could be successfully applied for diagnosing HNC patients by analyzing their exhaled breath. Consequently, the analysis of exhaled breath with an electronic nose offers various further possibilities for medical applications such as diagnosing and monitoring of other types of cancer and/ or other pulmonary diseases. 